\title{
Guidelines for Follow-Up of Women at High Risk for Inherited Breast Cancer: Consensus Statement from the Biomed 2 Demonstration Programme on Inherited Breast Cancer
}

\author{
P. Møller ${ }^{1, \#, ~ G . ~ E v a n s ~}{ }^{2}$, N. Haites ${ }^{3}$, \\ H. Vasen ${ }^{4}$, M.M. Reis ${ }^{5}$, E. Anderson ${ }^{6}$, \\ J. Apold ${ }^{7}$, S. Hodgson ${ }^{8}$, D. Eccles 9 , \\ H. Olsson ${ }^{10}$, D. Stoppa-Lyonnet ${ }^{11}$, \\ J. Chang-Claude ${ }^{12}$, P.J. Morrison ${ }^{13}$, \\ G. Bevilacqua $^{14}$, K. Heimdal ${ }^{1}$, L. Mæhle ${ }^{1}$, \\ F. Lalloo ${ }^{2}$, H. Gregory ${ }^{3}$, P. Preece ${ }^{5}$, \\ A. Borg ${ }^{10}$, N.C. Nevin ${ }^{13}$, M. Caligo ${ }^{14}$ \\ and C.M. Steel ${ }^{6}$ \\ ${ }^{1}$ Unit of Medical Genetics, The Norwegian \\ Radium Hospital, N-0310 Oslo, Norway \\ ${ }^{2}$ Family History Clinic, Centre for Cancer \\ Epidemiology, Christie Hospital NHS Trust, \\ Withington, Manchester M20 4QL, UK \\ ${ }^{3}$ Department of Medical Genetics, University \\ of Aberdeen, Polworth Building, Foresterhill, \\ Aberdeen AB25 2ZD, Scotland, UK \\ ${ }^{4}$ The Netherlands Foundation for the \\ Detection of Hereditary Tumours, c/o \\ University Hospital, Rijnsburgerweg 10, \\ Building no 5, 2333 AA Leiden, \\ The Netherlands \\ ${ }^{5}$ Departments of Surgery and Genetics, \\ Ninewells Hospital and Medical School, \\ Dundee DD1 9SY, Scotland, UK \\ ${ }^{6}$ Family History Clinic, Breast Screening \\ Centre, Ardmillan Terrace, Edinburgh EH11 \\ 2JL, Scotland, UK
}

\footnotetext{
\# Correspondence: Pål Møller, Unit of Medical Genetics, The Norwegian Radium Hospital, N-0310 Oslo, Norway, Tel.: + 47229 35675; Fax: + 47229 35219; Email: pmoller@ulrik.uio.no
}

\author{
${ }^{7}$ Department of Medical Genetics, Haukeland \\ University Hospital, N-5521 Bergen, Norway \\ ${ }^{8}$ Division of Medical and Molecular \\ Genetics, Guy's Hospital, London Bridge, \\ London SE1 9RT, UK \\ ${ }^{9}$ Department of Human Genetics, Princess \\ Ann Hospital, Southampton SO16 5YA, UK \\ ${ }^{10}$ Department of Oncology, University \\ Hospital, SE-221 85 Lund, Sweden \\ ${ }^{11}$ Unité de Génétique Oncologique, Institut \\ Curie, Section Medicale, 26, Rue d'Ulm \\ 75231 Paris Cedex 05, France \\ ${ }^{12}$ Division of Epidemiology, German Cancer \\ Research Centre, Im Neuenheimer Feld 280, \\ D-69120 Heidelberg, Germany \\ ${ }^{13}$ Northern Ireland Regional Medical \\ Genetics Centre, Belfast City Hospital Trust, \\ Belfast, BT9 7AB, UK \\ ${ }^{14}$ Institute of Pathology, University of Pisa, \\ via Roma 57, 56126 Pisa, Italy
}

\begin{abstract}
Protocols for activity aiming at early diagnosis and treatment of inherited breast or breastovarian cancer have been reported. Available reports on outcome of such programmes are considered here. It is concluded that the ongoing activities should continue with minor modifications. Direct evidence of a survival benefit from breast and ovarian screening is not yet available. On the basis of expert opinion and preliminary results from intervention programmes indicating good detection rates for early breast cancers and 5-year survival concordant with early diagnosis, we propose that women at high risk for inherited breast cancer be offered genetic counselling, education in 'breast awareness' and annual mammography and
\end{abstract}


clinical expert examination from around 30 years of age. Mammography every second year may be sufficient from 60 years on. BRCA1 mutation carriers may benefit from more frequent examinations and cancer risk may be reduced by oophorectomy before 40-50 years of age. We strongly advocate that all activities should be organized as multicentre studies subjected to continuous evaluation to measure the effects of the interventions on long-term mortality, to match management options more precisely to individual risks and to prepare the ground for studies on chemoprevention.

The Biomed 2 Demonstration Programme on Inherited Breast Cancer has compiled the results of the ongoing prospective studies, leading to the first report on outcome of early diagnosis and treatment of inherited breast cancer evaluated by survival after diagnosis [18]. 5-year event-free survival after diagnosis is $86 \%$, which is substantially better than all retrospective reports on prognosis of inherited breast cancer diagnosed outside specific follow-up programmes. Retrospective reports on age at onset of inherited breast cancer $[3,5,10]$ were verified by the prospective studies. It was demonstrated that, in families ascertained through clinical criteria, new breast cancers arise at a greatly increased rate (compared to the general population) in women aged 30 years or more $[13,15,17]$, and it was verified that, in the subgroup of breast-ovarian cancer kindreds, the majority of such cancers occur in BRCA1 mutation carriers [1]. It was found that uptake of genetic testing in kindred with demonstrated BRCA1 mutations can be as high as $80 \%$ without measurable negative effects on quality of life [22]. Different centres made similar use of cytology/biopsy, resulting in a diagnosis of cancer in $11-17 \%$ of all such procedures [19]. Comprehensive economic evaluation resulted in an estimated cost of Euro 750-1600 per life year gained through early diagnosis and treatment [11].

In BRCA1 mutation carriers, breast cancer tends to be diagnosed as infiltrating estrogen receptor negative cancers with histopathological signs of unfavorable prognosis $[1,12,14,23]$. It is not demonstrated, but it is suggested, that this subgroup may benefit from more frequent follow-up examinations.

Prophylactic mastectomy is discussed separately [9]. Results of tamoxifen chemoprevention trials to avoid breast cancer are inconclusive. Prophylactic oophorectomy may reduce breast cancer risk in BRCA1 mutation carriers, and use of hormone replacement therapy may not negate this effect [21]. Ongoing studies on the risk of contralateral cancers in BRCA1/2 mutation carriers, will indicate whether or not tamoxifen and/or chemotherapy may prevent breast cancers in mutation carriers. Oral contraceptive use reduces the risk of inherited ovarian cancer [20], but may not be recommended until the potential effect of increasing the breast cancer risk has been evaluated. Insurance issues related to predictive testing are discussed separately [16]. Modalities for treatment of demonstrated inherited breast cancer are discussed separately [7].

Mutations in BRCA1 and BRCA2 are associated with substantial risk of ovarian cancer. Retrospective studies indicate that ovarian cancer in BRCA1 mutation carriers occurs from 40 years of age on [5], while the risk is lower and the onset tends to be later in BRCA2 mutation carriers [10]. Prospective series confirm that breast cancer kindreds with two or more ovarian cancers, and/or cases with both breast and ovarian cancers, were likely to be caused by BRCA1 mutations, that ovarian cancers prospectively detected were rarely seen before 40 years of age, and that almost all ovarian cancers occur in BRCA1 mutation carriers [1,4]. No study on early diagnosis of inherited ovarian cancer has demonstrated safety: conversely, experience in several centres indicates an unfavourable prognosis for prospectively diagnosed cancers. Formal survival analyses from these series are not known to us. Infiltrating cancer has been found in ovaries prophylactically removed from patients declared to be disease-free by the operating gynaecologist [2]. Although a limited number of peritoneal carcinomatoses histopathologically classified as ovarian cancers have been reported after prophylactic oophorectomy, the preventive effect of prophylactic oophorectomy 
is judged to be good.

Based on these observations we advocate that the ongoing health programmes in most countries may continue essentially as they are carried out today $[6,8,24]$ and with modifications as indicated below.

\section{SUGGESTED GUIDELINES}

\section{Definition of women at high risk for inherited breast cancer}

- A family history of two or more first degree relatives (or second degree relatives though males) with early onset ( $<50$ years) breast cancer, and/or

- Multiple cases of breast cancers in the same lineage compatible with dominant inheritance in the family, and/or

- A combination of early onset breast cancer and ovarian cancer in the family, and/or

- Li Fraumeni syndrome (SBLA) or Cowden disease family, and/or

- Demonstrated BRCA1/2 truncating mutation or TP53 or PTEN mutation.

- Males with BRCA2 truncating mutation are also considered to be at risk.

\section{Genetic counselling}

Each woman at risk of inherited breast cancer should be entitled to genetic counselling by a specially trained doctor or genetic counsellor after validation of the family history and before risk estimates are finalized. The need for specially designed health care programmes should be determined and discussed in the course of such counselling. Predictive genetic testing should be carried out under strict quality control, with pre- and post-test genetic counselling by the responsible medical geneticist or associated genetic counsellor, and respecting the privacy and individual rights of each single patient. (Guidelines for these activities and the complicated problems related to sharing of family information between family members, are discussed separately.) Demonstrated high risk for inherited breast cancer should ensure access to the health care programmes suggested below, pending direct evidence of a survival benefit but on the assumption that this will emerge from presymptomatic detection of breast cancers.

\section{Follow-up examinations}

- Annual mammography and clinical expert examinations from 30 years of age (some centres modify starting age according to age of onset of disease in affected relatives). From 60 years of age mammography every second year (screening mammography) may be sufficient. Outside very high-risk families (BRCA1/2 carriers or clearly dominantly inherited disease in the kindred) more limited screening such as annual 35-50 years and 18 monthly to 60 years may be appropriate.

- BRCA1 mutation carriers (demonstrated or assumed by family history of two or more ovarian cancers and/or one relative with both breast and ovarian cancer) may benefit from mammography and clinical expert examinations twice a year and, where facilities permit, this option should be evaluated.

- Monthly self-examination or "breast awareness" education should be encouraged.

\section{Prophylactic surgery}

- BRCA2 mutation carriers (demonstrated, or strongly indicated by male breast cancer, plus other relevant cancers, in the family) may benefit from oophorectomy at menopause (4550 years of age)

- BRCA1 mutation carriers (demonstrated or strongly indicated by two or more ovarian cancers and/or one with both breast and ovarian cancer in the family) may benefit from oophorectomy at 35-50 years of age. Those not choosing oophorectomy should be offered follow-up including regular transvaginal ultrasound examination.

- Indications for prophylactic mastectomy are not considered here but should be subject to continuing evaluation. 


\section{Monitoring and evaluation of activity}

To evaluate the effect of screening and preventive activities, it is of the utmost importance that they are carried out as prospective multicentre studies subjected to strict protocols and continuous evaluation. This is essential not only to establish the effect of such interventions, but also to generate data from which screening and/or intervention strategies can be matched more precisely to individual estimates of risk and to provide the background necessary for assessment of new chemopreventive modalities in the near future.

\section{References}

[1] Borg, Å., Dørum, A., Heimdal, K. et al. BRCA1 $1675 \mathrm{delA}$ and $1135 \mathrm{ins} \mathrm{A}$ account for one third of Norwegian familial breast-ovarian cancer and are associated with later disease onset than less frequent mutations. Dis. Markers 15, (1999) 7984.

[2] Chen, K.T., Schooley, J.L. and Flam, M.S. Peritoneal carcinomatosis after prohpylactic oophorectomy in familial ovarian cancer syndrome. Obstet. Gynecol. 66(suppl 3), (1985) 93S-94S.

[3] Dørum, A., Heimdal, K., Hovig, E., Inganäs, M. and Møller, P. Penetrances of BRCA1 1675delA and 1135insA with respect to breast cancer and ovarian cancer. Am. J. Hum. Genet. 65, (1999) 671-679.

[4] Dørum, A., Heimdal, K., Løvslett, K. et al. Prospectively detected cancer in familial breast/ ovarian cancer screening. Acta Obst. Gyn. Scand. (in press).

[5] Easton, D.F., Ford, D., Bishop, D.T. and the Breast Cancer Linkage Consortium. Genetic heterogeneity and penetrance analysis of the BRCA1 and BRCA2 genes in breast cancer families. Am. J. Hum. Genet. 56, (1995) 256271.

[6] Eccles, D.M., Evans, D.G.R. and the UK Cancer Family Study Group. Guidelines for managing women with a family history of breast cancer. (in preparation).

[7] Eccles, D., Simmonds, P., Goddard, J., Coultas, M., Lalloo, F., Evans, G., Haites, N. and the European Familial Breast Cancer Collaborative
Group. Management of hereditary breast cancer. Dis. Markers 15, (1999) 187-189.

[8] Eisinger, F., Alby, N., Bremond, A. et al. Recommendations for management of hereditary breast and ovarian cancer: The French National ad hoc Committee. Ann. Oncol. 9, (1998) 939950.

[9] Evans, D.G.R., Anderson, E., Lalloo, F. et al. Utilisation of prophylactic mastectomy in 10 European centres. Dis. Markers 15, (1999) 148151.

[10] Ford, D., Easton, D.F., Stratton, M. and the Breast Cancer Linkage Consortium. Genetic heterogeneity and penetrance analysis of the BRCA1 and BRCA2 genes in breast cancer families. Am. J. Hum. Genet. 62, (1998) 676689.

[11] Heimdal, K., Mæhle, L. and Møller, P. Costs and benefits of diagnosing familial breast cancer. Dis. Markers 15, (1999) 167-173.

[12] Johannsson, O.T., Idvall, I., Anderson, C. et al. Tumor biological features of BRCA1-induced breast and ovarian cancer. Eur. J. Cancer 33, (1997) 362-371.

[13] Kollias, J., Sibbering, D.M., Blamey, R.W. et al. Screening women aged less than 50 years with a family history of breast cancer. Eur. J. Cancer 34, (1998) 878-883.

[14] Lakhani, S.R. et al. Multifactorial analysis of differences between sporadic breast cancers and cancers involving BRCA1 and BRCA2 mutations. J. Natl. Cancer Inst. 90(15), (1998) 1138-1145.

[15] Lalloo, F., Boggis, C.R.M., Evans, D.G.R., Shenton, A., Threlfall, A.G. and Howell, A. Screening by mammography, women with a family history of breast cancer. Eur. J. Cancer 34, (1998) 937-940.

[16] Morrison, P.J., Steel, C.M., Vasen, H.F.A. et al. Insurance implications for individuals with a high risk of breast and ovarian cancer in Europe. Dis. Markers 15, (1999) 159-165.

[17] Møller, P., Maehle, L., Heimdal, K. et al. Prospective findings in breast cancer kindreds: annual incidence rates according to age, stage at diagnosis, mean sojourn time, and incidence rates for contralateral cancer. The Breast 7, (1998) 5559.

[18] Møller, P., Reis, M.M., Evans, G. et al. Efficacy of early diagnosis and treatment in women with a family history of breast cancer. Dis. Markers 15, (1999) 179-186.

[19] Møller, P., Evans, G., Anderson, E. et al. Use of 
cytology to diagnose inherited breast cancer. Dis. Markers 15, 1999206.

[20] Narod, S.A., Risch, H., Moslehi, R. et al. Oral contraceptive and the risk of hereditary ovarian cancer. N. Engl. J. Med. 339, (1998) 424-428.

[21] Rebbeck, T.R., Levin, A.M., Eisen, A. et al. Breast cancer risk after bilateral prophylactic oophorectyomy in BRCA1 mutation carriers. $J$. Natl. Cancer Inst. 91, (1999) 1475-1479.

[22] Reichelt, J.G., Dahl, A.A., Heimdal, K. and Møller, P. Uptake to genetic testing and pre-test levels of mental distress in Norwegian families with known BRCA1 mutations. Dis. Markers 15, (1999) 139-143.

[23] Sobol, H., Eisinger, F., Stoppa-Lyonnet, D.,
Longy, M., Jacquemier, J., Birnbaum. Histoprognostic grade in hereditary breast cancer: is inheritance linked to BRCA1 a bad prognostic factor? In: Hereditary cancer. Müller, H., Scott, R.J., Weber, W. (Eds.) 2nd int. res. conf. on familial cancer, Basel 1995. Karger, Basle (1996) pp 11-18.

[24] Vasen, H.F., Haites, N.E., Evans, D.G. and the European Familial Breast Cancer Collaborative Group. Current policies for surveillance and management in women at risk of breast and ovarian cancer: a survey among 16 European family cancer clinics. Eur. J. Cancer 34, (1998) 1922-1926. 


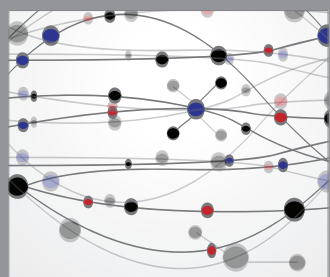

The Scientific World Journal
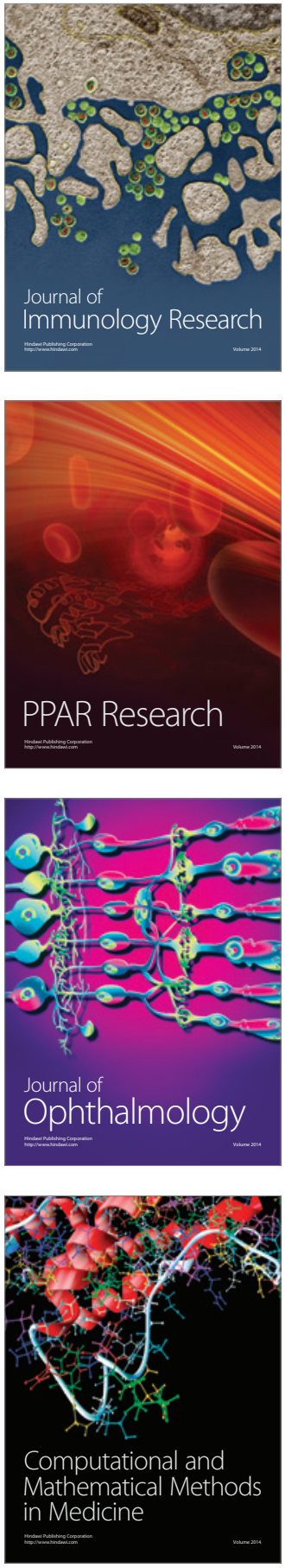

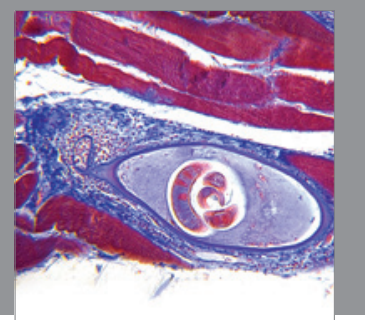

Gastroenterology

Research and Practice
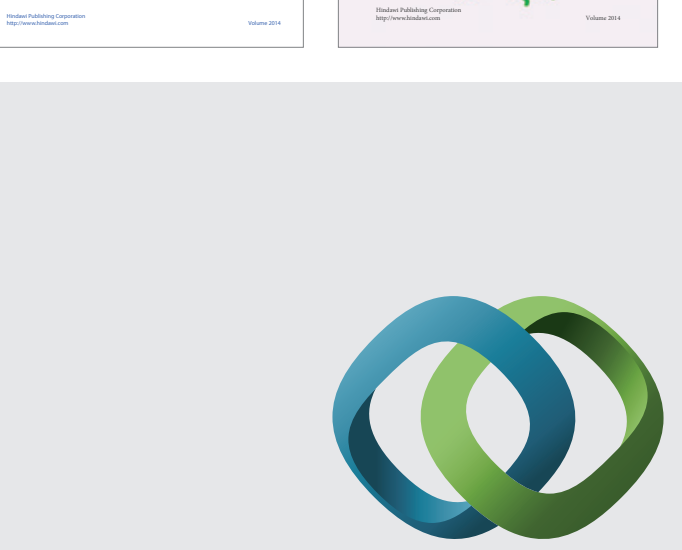

\section{Hindawi}

Submit your manuscripts at

http://www.hindawi.com
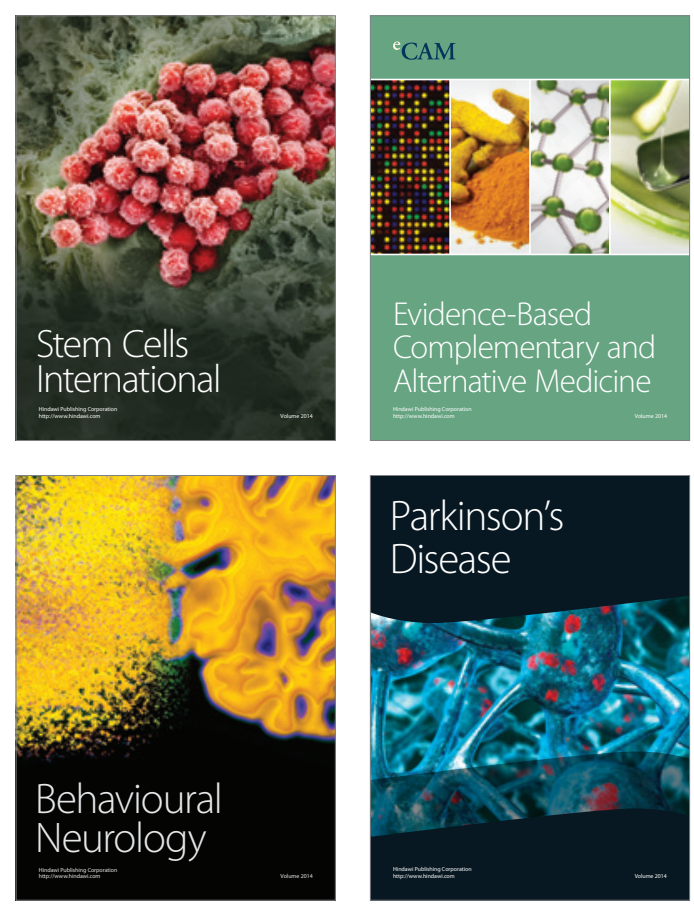

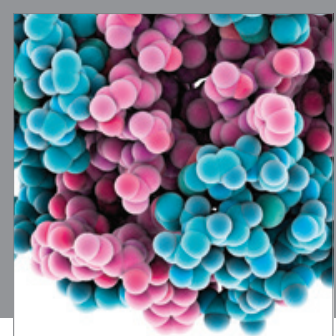

Journal of
Diabetes Research

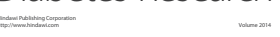

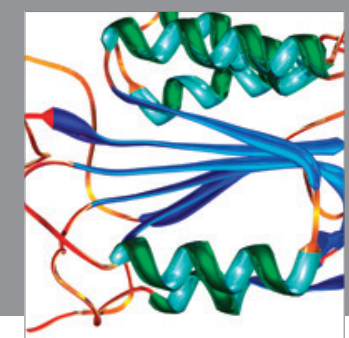

Disease Markers
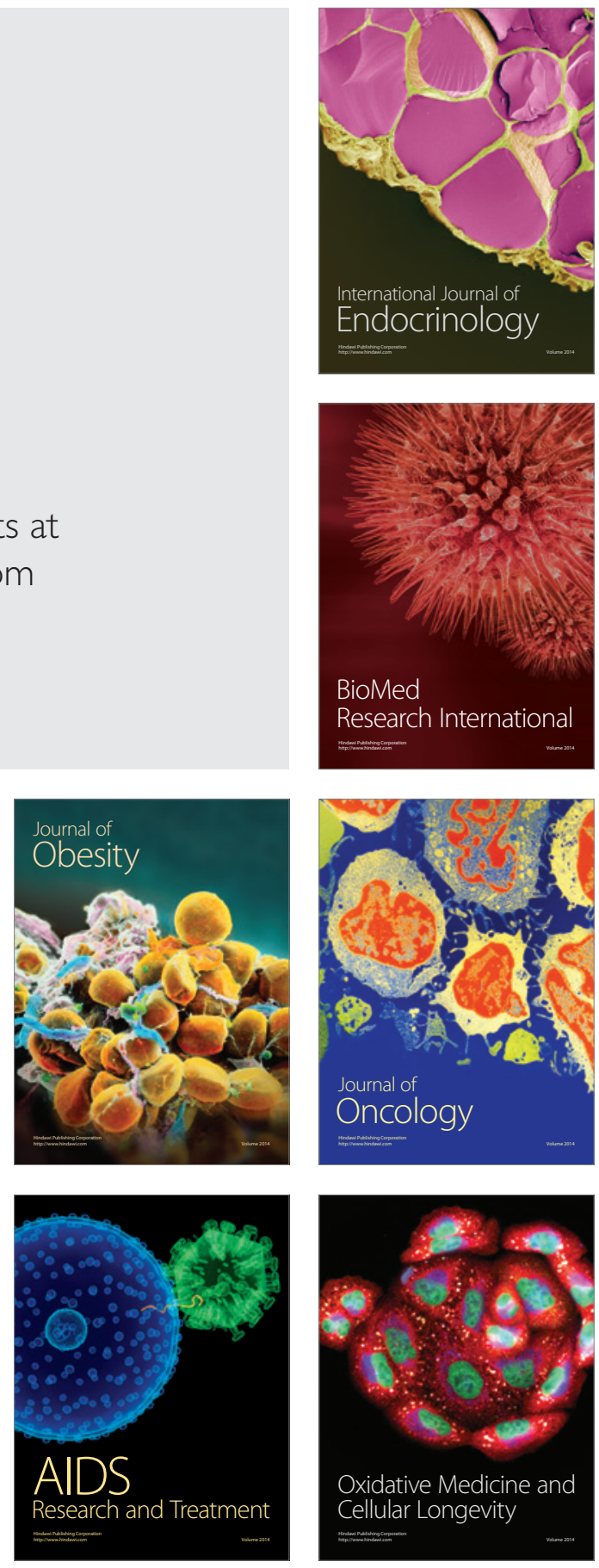